\title{
Identification of Two Novel VHR Phosphatase Inhibitors with Structure-Based Virtual Screening
}

\author{
Hwangseo Park, ${ }^{\mathrm{a}, *}$ Jeong-Yi Jeon, ${ }^{\dagger, \mathrm{a}}$ Dae-Gwin Jeong, ${ }^{\S}$ and Seong Eon Ryu ${ }^{\dagger, \star, *}$ \\ Department of Bioscience and Biotechnology, Sejong University, Seoul 143-747, Korea. *E-mail: hspark@sejong.ac.kr \\ ${ }^{\dagger}$ Department of Bioengineering and $\$$ Institute for Bioengineering and Biopharmaceutical Research, \\ Hanyang University, Seoul 133-791, Korea. E-mail: ryuse@hanyang.ac.kr \\ ${ }^{\S}$ Medical Proteomics Research Center, KRIBB, 52 Euheun-dong, Yusong-gu, Daejeon 305-333, Korea \\ Received September 9, 2010, Accepted September 29, 2010
}

Key Words: VHR phosphatase, Virtual screening, Inhibitor, Docking, Solvation

Protein tyrosine phosphatases (PTPs) are a family of the regulatory enzymes that are responsible for the dephosphorylation of phosphotyrosine residues in the protein substrates. A series of experimental evidence has been reported so far to support the correlation between malfunctions in PTP activity and various diseases including cancer, neurological disorders, and diabetes. ${ }^{1}$ This has made PTPs be a promising target for drug discovery. Of the various PTPs, vaccinia H1-related (VHR) phosphatase belongs to a dual-specificity phosphatase (DSP) and dephosphorylates the activated ERK and JNK MAP kinases, which has an effect of weakening the ERK signaling cascade in mammalian cells. ${ }^{2,3}$

It has been known that the human VHR phosphatase would be involved in the regulation of cell-cycle progression and modulated during the cell cycle. ${ }^{4}$ Also, the cells lacking VHR were shown to be arrested at the G1-S and G2-M transitions of the cell cycle with a decreased telomerase activity. This indicates that VHR can serve as a therapeutic target for cancer. Furthermore, the VHR activity has been known to related with the immune response of the activated $\mathrm{T}$ cells ${ }^{5}$ and vaccinia-related kinase 3 (VRK3). ${ }^{6}$ The involvement of VHR in immune response further motivates the discovery of small-molecule ligands to regulate its activity.

A conserved structural scaffold for both DSPs and PTPs was observed in the X-ray crystal structure of VHR. ${ }^{7}$ Its shallow active site pocket is consistent with the broad substrate specificity for phosphorylated serine, threonine, and tyrosine residues. The presence of the positively charged crevices near the active site can be invoked to explain the preference of VHR for the substrates with two phosphorylated residues. The catalytic residue (Cys124) resides in close proximity to the central sulfur atom of the substrate analogue in the crystal structure at a distance of $3.65 \AA$. This implies that Cys 124 acts as a nucleophile attacking the central phosphorus atom of a substrate to form a phosphoenzyme intermediate. ${ }^{8}$ It was also shown in the X-ray crystal structure that the substrate analogue should be stabilized in the active site through the formation of multiple hydrogen bonds with Arg130. These structural features provide useful information for designing the small-molecules inhibitors of VHR. However, only a few classes of VHR inhibitors have been discovered so far. ${ }^{9-15}$

\footnotetext{
${ }^{\mathrm{a}}$ These two authors contribute equally to this work.
}

In the present study, we identify two novel inhibitors of VHR phosphatase based on the structure-based drug design protocol involving the virtual screening with docking simulations and in vitro enzyme assay. Virtual screening with docking simulation has not always been successful due to the inaccuracy in the scoring function. ${ }^{16}$ The characteristic feature that disciriminates our virtual screening approach from the others lies in the implementation of an accurate solvation model in calculating the binding free energy between VHR and its putative ligands, which would have an effect of increasing the hit rate in enzyme assay. ${ }^{17,18}$ Indeed, the inclusion of the desolvation energy term in the scoring function can diminish the overestimation of the binding affinity of a ligand with many polar atoms. It will be shown that the docking simulation with the improved scoring function can be a useful for elucidating the activities of the identified inhibitors, as well as for enriching the chemical library with molecules that are likely to have inhibitory activities.

Of the 85,000 compounds subject to the virtual screening with docking simulations, 100 top-scored compounds were selected as virtual hits. 89 of them were available from the compound supplier and were tested for inhibitory activity against VHR by in vitro enzyme assay. The inhibition assay was performed in duplicates at all concentrations of the inhibitors and the average values were used as data points. As a result, we identified 2 compounds that inhibited the catalytic activity of VHR by more than $50 \%$ at the concentration of $50 \mu \mathrm{M}$. The chemical structures and the inhibitory activities of the newly identified inhibitors are shown in Figure 1 and Table 1, respectively. We note that compounds $\mathbf{1}$ and $\mathbf{2}$ possess a benzoic acid group at the end of the molecular structure. The calculated binding free energies of these two potent inhibitors are also similar: 26.1 and 24.9 $\mathrm{kcal} / \mathrm{mol}$ for $\mathbf{1}$ and $\mathbf{2}$, respectively. To the best of our knowledge, neither of these compounds has been reported as VHR inhibitors so far. Judging from the potency and the structural difference, both of the newly identified inhibitors can serve as a new inhibitor scaffold for further development by structure-activity relationship (SAR) methods to optimize the inhibitory activities.

To obtain some energetic and structural insight into the inhibitory mechanisms by the two identified inhibitors of VHR, their binding modes in the active site were investigated using the AutoDock program. The calculated binding mode of $\mathbf{1}$ in the active site of VHR is shown in Figure 2. It is seen that the 
Table 1. Inhibitory activities of $\mathbf{1}$ and $\mathbf{2}$ and against VHR phosphatase

\begin{tabular}{cc}
\hline compound & $\mathrm{IC}_{50}(\mu \mathrm{M})$ \\
\hline $\mathbf{1}$ & $1.7 \pm 0.4$ \\
$\mathbf{2}$ & $18.7 \pm 2.6$ \\
\hline
\end{tabular}

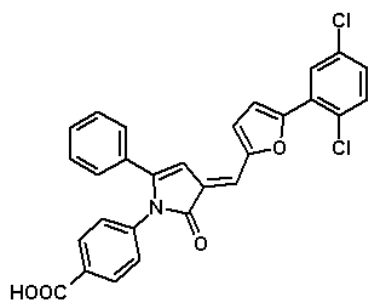

1

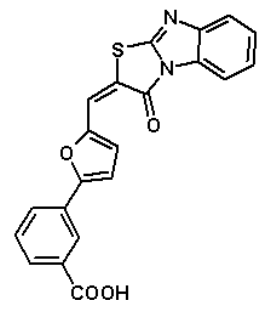

Figure 1. Chemical structures of the newly identified VHR phosphatase inhibitors.

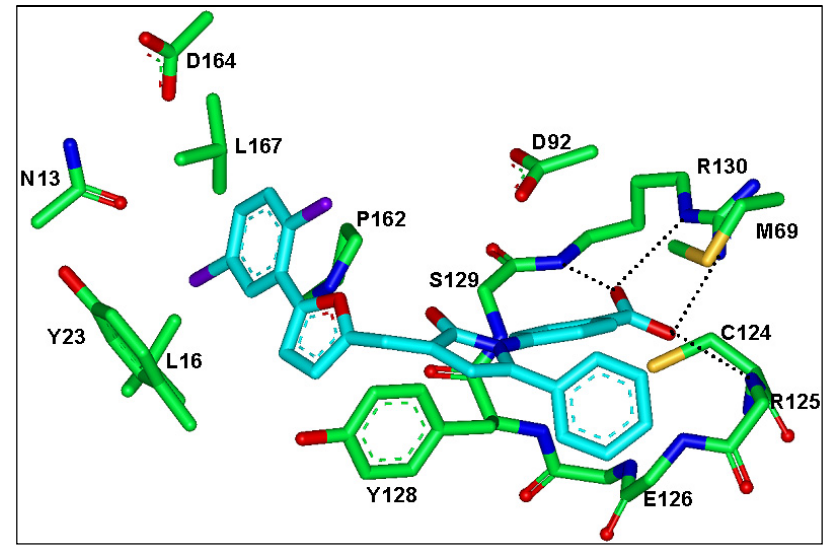

Figure 2. Binding mode of $\mathbf{1}$ in the active site of VHR phosphatase. Carbon atoms of the protein and the ligand are indicated in green and cyan, respectively. Each dotted line indicates a hydrogen bond.

carboxylate group of the inhibitor points toward the catalytic residue Cys 124 at a distance of $3-4 \AA$. We also note that one of the carboxylate oxygens receives two hydrogen bonds from the backbone amide group and the side chain NE atom of Arg130. The other carboxylate oxygen also forms the bifurcated hydrogen bonds with the side-chain guanidium group of Arg130 and backbone amide group of Arg125. Because the carboxylate group of 1 resides in a close proximity to Cys 124 and is stabilized by the formation of multiple hydrogen bonds at the active site, the benzoate moiety seems to be an effective surrogate for the substrate phosphate group that binds tightly in the active site. The inhibitor $\mathbf{1}$ can be further stabilized by the hydrophobic interactions of its aromatic groups with the side chains of Leu16, Tyr23, Tyr128, Pro162, and Leu167. Judging from the structural features of the calculated VHR-1 complex, the micromolar inhibitory activity of $\mathbf{1}$ seems to stem from the establishments of multiple hydrogen bonds and hydrophobic interactions in the active site in a simultaneous fashion.

Figure 3 shows the most stable binding mode of $\mathbf{2}$ in the active site of VHR obtained from docking simulations. The binding mode of $\mathbf{2}$ is similar to that of $\mathbf{1}$ in that the benzoate group points

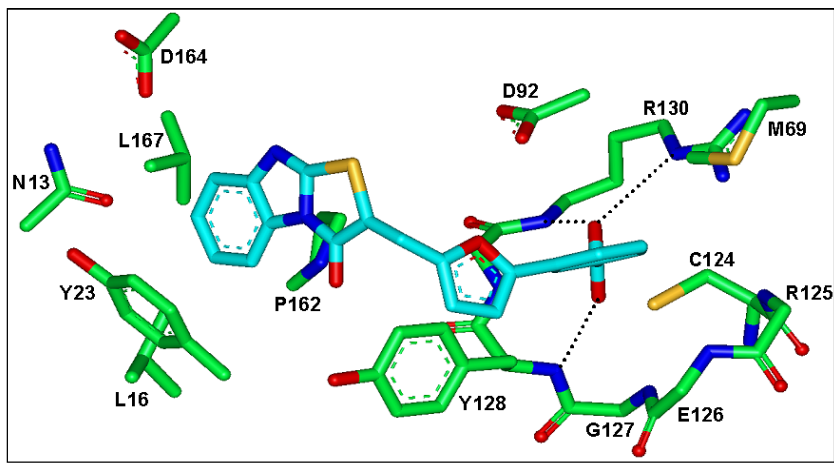

Figure 3. Binding mode of $\mathbf{2}$ in the active site of VHR phosphatase. Carbon atoms of the protein and the ligand are indicated in green and cyan, respectively. Each dotted line indicates a hydrogen bond.

toward the catalytic residue Cys 124 with the terminal carboxylate group being stabilized by four hydrogen bonds with the backbone amide groups and the side chain of Arg130 in the active site. However, the number of hydrogen bonds decreases from four to three in going from VHR-1 to VHR-2 complexes, which is likely to have an effect of lowering the inhibitory activity of $\mathbf{2}$. As in the VHR-1 complex, the hydrophobic interactions seem also to be a significant binding force for stabilizing $\mathbf{2}$ in the active site of VHR because its aromatic rings form van der Waals contacts with the side chains of Leu16, Tyr23, Tyr128, Pro162, and Leu167. Because the hydrophobic interactions are established in a similar fashion in the two enzyme-inhibitor complexes, the decrease in the number of hydrogen bonds should be responsible for the lower inhibitory activity of $\mathbf{2}$ than $\mathbf{1}$.

In summary, we have identified two new novel inhibitors of VHR phosphatase by means of the structure-based virtual screening with docking simulations. These inhibitors exhibit a significant potency with $\mathrm{IC}_{50}$ values of 1.7 and $18.7 \mu \mathrm{M}$ and have a benzoate group as an effective surrogate for the phosphate group in the substrate. Considering the novelty and the potency, each of the newly discovered inhibitor deserves consideration for further development by SAR studies. Detailed binding mode analyses with docking simulation indicate that the inhibitors can be stabilized in the active site by the simultaneous establishment of the multiple hydrogen bonds and van der Waals contacts.

\section{Experimental Section}

Virtual screening of VHR inhibitors. The X-ray crystal structure of VHR phosphatase in complex with a substrate analogue (PDB code: 1VHR $)^{7}$ was selected as the receptor model in the virtual screening with docking simulations. After removing the ligand and solvent molecules, hydrogen atoms were added to each protein atom. A special attention was paid to assign the protonation states of the ionizable Asp, Glu, His, and Lys residues. The side chains of Asp and Glu residues were assumed to be neutral if one of their carboxylate oxygens pointed toward a hydrogen-bond accepting group including the backbone aminocarbonyl oxygen at a distance within $3.5 \AA$, a generally accepted distance limit for a hydrogen bond of moderate strength. Si- 
milarly, the lysine side chains were assumed to be protonated unless the NZ atom was in proximity of a hydrogen-bond donating group. The same procedure was also applied to determine the protonation states of ND and NE atoms in His residues.

The docking library for VHR comprising about 85,000 compounds was constructed from the latest version of the chemical database distributed by Interbioscreen (http://www.ibscreen. com) containing approximately 30,000 natural and 320,000 synthetic compounds. The selection was based on the drug-like filters that adopt only the compounds with physicochemical properties of potential drug candidates ${ }^{17}$ and without reactive functional group(s). All of the compounds included in the docking library were then subjected to the Corina program to generate their three-dimensional atomic coordinates, followed by the assignment of Gasteiger-Marsilli atomic charges. ${ }^{18} \mathrm{We}$ used the AutoDock program ${ }^{19}$ in the virtual screening of VHR inhibitors because the outperformance of its scoring function over those of the others had been shown in several target proteins. $^{20,21}$ AMBER force field parameters were assigned for calculating the van der Waals interactions and the internal energy of a ligand as implemented in the AutoDock program.

In vitro enzyme assay. VHR phosphatase was subcloned into pET28a and overexpressed using Escherichia coli BL21(DE3) strain. Cells were grown at $291 \mathrm{~K}$ after induction with $0.1 \mathrm{mM}$ IPTG for 12 hours. His-tagged VHR was purified by nickelaffinity chromatography and dialyzed against buffer containing $20 \mathrm{mM}$ Tris- $\mathrm{HCl}$ (pH 8.0), 0.2 M NaCl, and 5 mM DTT. 89 compounds selected from the precedent virtual screening were evaluated for their in vitro inhibitory activity against the recombinant human VHR. Assays were performed by monitoring the extent of hydrolysis of 6,8-difluoro-4-methyl-umbelliferyl phosphate (DiFMUP) with a spectrofluorometric assay. The purified VHR (5 nM), DiFMUP $(10 \mu \mathrm{M})$, and a candidate inhibitor were incubated in the reaction mixture containing $20 \mathrm{mM}$ Tris- $\mathrm{HCl}$ (pH 8.0), 0.01\% Trition X-100, and 5mM DTT for 20 minutes. This enzymatic reaction was stopped with the addition of sodium orthovanadate $(1 \mathrm{mM})$. The phosphatase activities were then checked by the absorbance changes due to the hydrolysis of the substrate at $460 \mathrm{~nm}$. $\mathrm{IC}_{50}$ values of the inhibitors were determined from direct regression curve analysis.
Acknowledgments. This work was supported by the grant from Hanyang University (Grant No. 20100244).

\section{References}

1. Waldmann, H.; Bialy, L. Angew. Chem. Int. Ed. 2005, 44, 3814.

2. Alonso, A.; Saxena, M.; Williams, S.; Mustelin, T. J. Biol. Chem. 2001, 276, 4766.

3. Todd, J. L.; Tanner, K. G.; Denu, J. M. J. Biol. Chem. 1999, 274, 13271.

4. Rahmouni, S.; Cerignoli, F.; Alonso, A.; Tsutji, T.; Henkens, R.; Zhu, C.; Louis-dit-Sully, C.; Moutschen, M.; Jiang, W.; Mustelin, T. Nat. Cell Biol. 2006, 8, 524.

5. Alonso, A.; Rahmouni, S.; Williams, S.; van Stipdonk, M.; Jaroszewski, L.; Godzik, A.; Abraham, R. T.; Schoenberger, S. P.; Mustelin, T. Nat. Immunol. 2003, 4, 44.

6. Kang, T. H.; Kim, K. T. Nat. Cell Biol. 2006, 8, 863.

7. Yuvaniyama, J.; Denu, J. M.; Dixon, J. E.; Saper, M. A. Science 1996, 272, 1328

8. Zhou, G.; Denu, J. M.; Wu, L.; Dixon, J. E. J. Biol. Chem. 1994, 269, 28084.

9. Bae, E. Y.; Oh, H.; Oh, W. K.; Kim, M. S.; Kim, B. S.; Kim, B. Y.; Sohn, C. B.; Osada, H.; Ahn, J. S. Planta Med. 2004, 70, 869.

10. Lee, M. S.; Oh, W. K.; Kim, B. Y.; Ahn, S. C.; Kang, D. O.; Sohn, C. B.; Osada, H.; Ahn, J. S. Planta Med. 2002, 68, 1063.

11. Ueda, K.; Usui, T.; Nakayama, H.; Ueki, M.; Takio, K.; Ubukata, M.; Osada, H. FEBS Lett. 2002, 525, 48.

12. Usui, T.; Kojima, S.; Kidokoro, S.; Ueda, K.; Osada, H.; Sodeoka, M. Chem. Biol. 2001, 8, 1209.

13. Sodeoka, M.; Sampe, R.; Kojima, S.; Baba, Y.; Usui, T.; Ueda, K.; Osada, H. J. Med. Chem. 2001, 44, 3216.

14. Lazo, J. S.; Nunes, R.; Skoko, J. J.; Queiroz de Oliveira, P. E.; Vogt, A.; Wipf, P. Bioorg. Med. Chem. 2006, 14, 5643.

15. Shi, Z.; Tabassum, S.; Jiang, W.; Zhang, J.; Mathur, S.; Wu, J.; Shi, Y. ChemBioChem 2007, 8, 2092.

16. Warren, G. L.; Andrews, C. W.; Capelli, A.-M.; Clarke, B.; LaLonde, J.; Lambert, M. H.; Lindvall, M.; Nevins, N.; Semus, S. F.; Senger, S.; Tedesco, G.; Wall, I. D.; Woolven, J. M.; Peishoff, C. E.; Head, M. S. J. Med. Chem. 2006, 49, 5912.

17. Lipinski, C. A.; Lombardo, F.; Dominy, B. W.; Feeney, P. J. Adv. Drug Delivery Rev. 1997, 23, 3.

18. Gasteiger, J.; Marsili, M. Tetrahedron 1980, 36, 3219.

19. Morris, G. M.; Goodsell, D. S.; Halliday, R. S.; Huey, R.; Hart, W. E.; Belew, R. K.; Olson, A. J. J. Comput. Chem. 1998, 19, 1639.

20. Park, J.-H.; Ko, S.; Park, H. Bull. Korean Chem. Soc. 2008, $29,921$.

21. Lee, W.; Park, H.; Lee, S. Bull. Korean Chem. Soc. 2008, 29, 363. 